J. Clin. Chem. Clin. Biochem.

Vol. 15,1977 , pp. $389-392$

\title{
Die Differenzierung von VLDL-Subfraktionen in der Diagnostik von Typ III Hyperlipoproteinämien ${ }^{1}$ )
}

\author{
Von $K . H$. Vogelberg \\ Klinische Abteilung des Diabetes-Forschungsinstitutes an der Universität Düsseldorf
}

(Eingegangen am 20. Oktober/16. Dezember 1976)

\begin{abstract}
Zusammenfassung: Hyperlipoproteinämien vom Typ III sind durch „floating“- $\beta$-Lipoproteine gekennzeichnet, die lipidelektrophoretisch als VLDL-Subfraktion ( $\beta$-VLDL) und lipidchemisch durch bestimmte Kriterien der TriglyceridCholesterinzusammensetzung des Gesamtserums bzw. der zugehörigen VLDL-Serumfraktion nachweisbar sein können. In der vorliegenden Arbeit wurde die diagnostische Wertigkeit dieser Kriterien mit dem für Typ III Hyperlipoproteinämien als spezifisch angenommenen E III-Peptidverlust der VLDL-Apolipoproteine verglichen. Die Untersuchungen zeigten, daß bei 42 Personen mit einer VLDL Subfraktion $7 \mathrm{mal}$ ein Verlust des Peptids nachweisbar war. Bei diesen Personen war der Cholesterin/Triglycerid Quotient des Gesamtserums 0,94 $\pm 0,45$ und jener der VLDL-Serumfraktion $0,59 \pm 0,25$. Der Quotient des VLDL-Cholesterin/Serumtriglyceridgehalt war $0,43 \pm 0,21$ und die elektrophoretische Mobilität der VLDL-Subfraktion 0,38 $\pm 0,04$. Die Untersuchung des VLDL-Cholesterin/Triglyceridquotienten erbrachte in 4 Fällen (10\%), der Quotient aus dem VLDL-Cholesterin/Serumtriglyceridgehalt in 10 Fällen (24\%) ein „falsch-positives“ Ergebnis. Dieser Vergleich zeigt, daß die untersuchten Kriterien zur Identifizierung von „floating“$\beta$-Lipoproteinen bzw. einer $\beta$-VLDL-Subfraktion nur als indirekte Hinweise einer Typ III Hyperlipoproteinämie bedeutsam sind.
\end{abstract}

\section{Differentiation of VLDL subfractions in the diagnosis of type III hyperlipoproteinaemias}

Summary: Type III hyperlipoproteinaemias are characterized by "floating" $\beta$-lipoproteins. In lipid electrophoresis these are present as a VLDL subfraction ( $\beta$-VLDL). They can also be detected chemically on the basis of the cholesterol/ triglyceride ratio of the serum or the corresponding VLDL serum fraction. In the present work, the diagnostic value of these ratios is compared with that of E III peptide loss from VLDL-apolipoproteins, which is thought to be specific for type III hyperlipoproteinaemias. Of 42 persons with a VLDL subfraction, seven showed a loss of the peptide. In these persons, the cholesterol/triglyceride quotient was $0.94 \pm 0.45$ for the whole serum, and $0.59 \pm 0.25$ for the serum VLDL fraction. The quotient for VLDL cholesterol/serum triglyceride was $0.43 \pm 0,21$, and the electrophoretic mobility of the VLDL subfraction was $0.38 \pm 0.04$. Investigation of the VLDL-cholesterol/triglyceride quotient gave four (10\%) false positives, while the quotient for VLDL-cholesterol/serum triglyceride gave ten (24\%) false positives. This comparison shows that the criteria used for identification of "floating" $\beta$-VLDL subfraction are only indirectly useful in the diagnosis of type III hyperlipoproteinaemia.

\section{Einführung}

Hyperlipoproteinämien vom Typ İi sind durch sog. “floating"- $\beta$-Lipoproteine ( $\beta$-VLDL) gekennzeichnet (1). Das Lipoproteinmuster dieser Fettstoffwechselstörungen kann differentialdiagnostisch durch lipidchemische $(2,3)$, immunologische (4), Präzipitations- oder Unterscheidungsmerkmale der Ultrazentrifugation $(5,6,7)$ von anderen Lipoproteinmustern abgegrenzt werden. Utermann et al.

\footnotetext{
1) Mit Unterstützung der Deutschen Forschungsgemeinschaft Bad Godesberg (SFB 113, A IIÍ-9).
}

(8) konnten zeigen, daß ,floating "- $\beta$-Lipoproteine wahrscheinlich auch durch den Verlust eines E-Peptids (Apolipoprotein E-III) der VLDL ${ }^{2}$ )-Apolipoproteine spezifisch nachweisbar sind. Nachfolgend wurde die Wertigkeit lipidchemischer und lipidelektrophoretischer Kriterien einer Typ III Hyperlipoproteinämie anhand dieses Peptid-Verlustes bei 42 Seren mit einer VLDL Subfraktion geprüft.

\footnotetext{
2) Abkürzungen: VLDL = „very löw density lipoproteins" LDL = "low density lipoproteins" HDL = "high density lipoproteins“"
} 


\section{Patienten und Untersuchungsmethoden}

Zur Differenzierung verschiedener VLDL-Subfraktionen wurden nacheinander 442 Seren von 217 Personen untersucht. Bei den Personen handelte es sich um stationär und/oder ambulant behandelte Patienten der Klinischen Abteilung des DiabetesForschungsinstituts Düsseldorf. Die Auswahl der Patienten richtete sich nach Vorhandensein und Qualität der Lipid-Elektrophorese und der Vollständigkeit zugehöriger Lipidbestimmungen im Nativserum bzw. definierten Serum-Lipoproteinfraktionen der präparativen Ultrazentrifugation (s. u.). Patienten unter 19 Jahren wurden von der Studie ausgeschlossen, ebenso Seren, deren Triglycerid- und Cholesteringehalt „normal“ war. Als obere Normgrenze galt eine Triglyceridkonzentration von 0,17 $\mathrm{mmol} / \mathrm{l}$ und eine Cholesterinkonzentration von $6,73 \mathrm{mmol} / 1$ Serum. Die angewandten Labormethoden wurden bereits früher beschrieben (9). Als Kriterium einer VLDL Subfraktion galt der elektrophoretische Nachweis einer VLDL-Doppelbande. Die VLDL-Apolipoproteinanalyse wurde von Dr. G. Utermann (Institut für Humangenetik, Universität Marburg) durchgeführt. Statistische Signifikanzberechnungen erfolgten im t-Test.

\section{Ergebnisse}

\section{Vorkommen}

Von den untersuchten 217 Personen wiesen 68 (31\%) lipidelektrophoretisch eine VLDL-Subfraktion auf. Es handelte sich um 41 Männer und 27 Frauen im Durchschnittsalter von $47 \pm 13$ Jahren (23-64 Jahren) bzw. $53 \pm 11$ Jahren (31-59 Jahren) (Tab. 1). Das Kontrollkollektiv wurde aus der restlichen Personengruppe entsprechend der Verteilung von Alter, Geschlecht, Körpergewicht und Glucosetoleranz des Patientenkollektivs ausgesucht. Die Lipidbestimmung der Serumlipoproteinfraktionen ergab, daß in Seren mit einer VLDL-Subfraktion die Cholesterinkonzentration in VLDL bzw. die Triglyceridkonzentration in LDL und HDL z. T. signifikant größer war als im Kontrollkollektiv.

\section{Differentialdiagnose}

VLDL-Subfraktionen konnten bei unterschiedlichen Lipoproteinmustern beobachtet werden (Abb. 1). Von 42 der untersuchten Seren wiesen in 2-9 Analysen 13 konstant eine VLDL-Subfraktion auf; in 29 Fällen war der Befund inkonstant. Die Konzentration bzw. Breite der Subfraktion war individuell variabel. Die elektrophoretische Mobilität schwankte zwischen 0,34 und 0,65 (bezogen auf die anodenwärts gelegene $a$-Lipoproteinbande).

Aufgrund von Literaturangaben wurde eine Hyperlipoproteinämie Typ III angenommen, wenn der VLDLCholesterin/VLDL-Triglycerid Quotient größer als 0,42 (10) und/oder der VLDL-Cholesterin/Serumtriglycerid Quotient größer als 0,30 (11) war. Die VLDLApolipoproteinanalyse ergab, daß bei 7 Seren mit einer VLDL-Subfraktion (16,7\%) ein Verlust des E-III Apolipoprotein-Peptids vorlag. Der VLDL-Cholesterin/ VLDL-Triglycerid Quotient stimmte in 4 $(9,5 \%)$ und der Quotient aus dem VLDL-Cholesterin/Serumtriglyceridgehalt in 10 Fällen $(23,8 \%)$ nicht mit dem Ergebnis der Apoproteinanalyse überein. „Falsch negative“ Ergebnisse der lipidchemischen Untersuchungen wurden nicht beobachtet.

\section{Diskussion}

Die vorliegenden Untersuchungen zeigen, daß Subfraktionen der VLDL-Lipoproteine keineswegs immer als „floating“ $\beta$-Lipoproteine bzw. $\beta$-VLDL anzusprechen sind. Der elektrophoretische Nachweis einer VLDL-Subfraktion und die untersuchten lipidchemischen Kriterien einer Typ III Hyperlipoproteinämie sind nur als indirekte

Tab. 1. Anzahl, Alter, relatives Körpergewicht (Broca-Index), orale Glucosetoleranz und bestimmte Lipidkonzentrationen bei Seren mit $(n=68)$ bzw. ohne $(n=68)$ lipidelektrophoretischen Nachweis einer VLDL-Subfraktion.

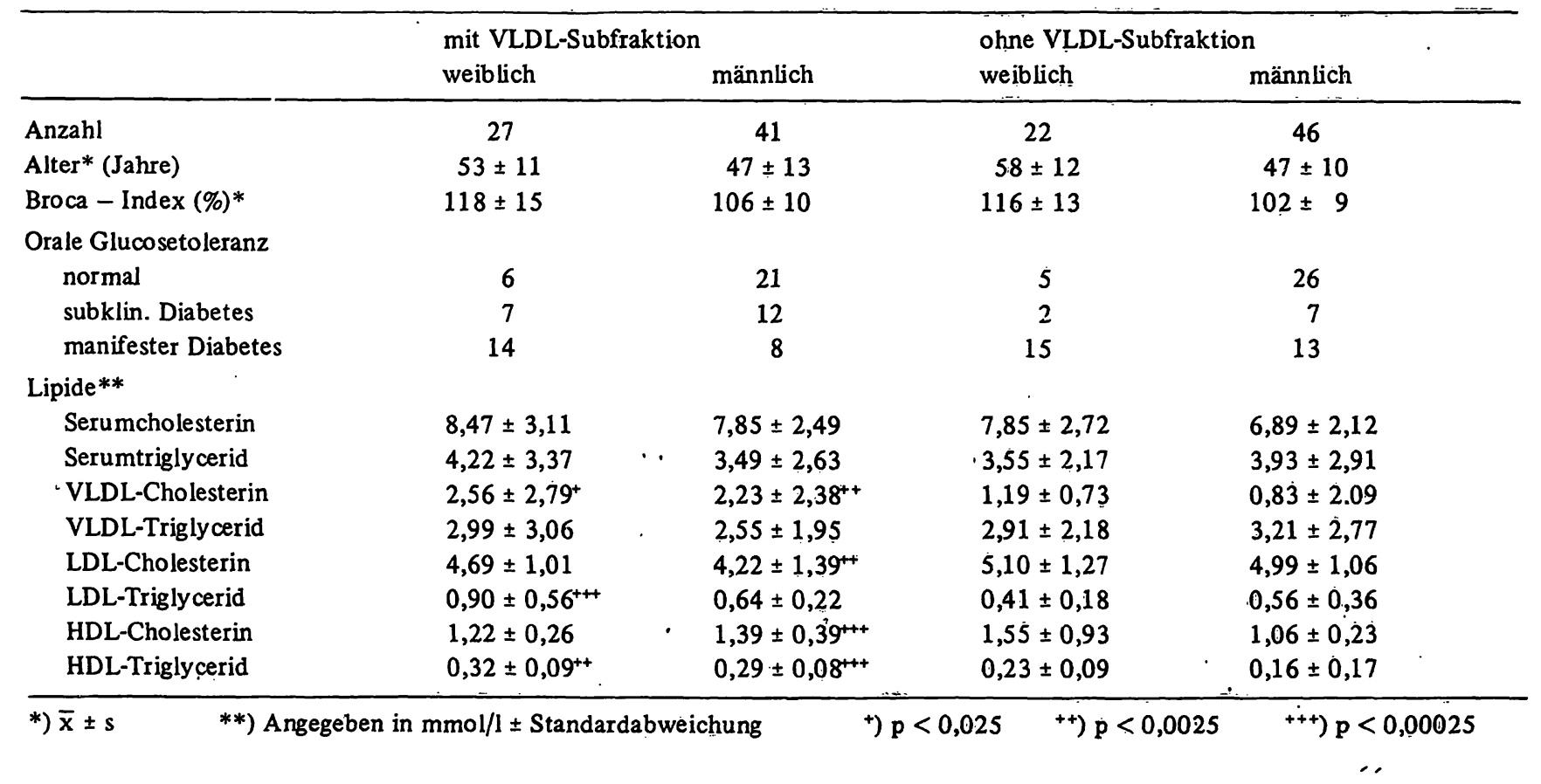


Abb. 1. VLDL-Subfraktionen bei verschiedenen Hyperlipoproteinämiemustern der Agarosegel-Lipidelektrophorese. Aufgetragen wurde jeweils das Nativserum und die Ultrazentrifugen-Serumfraktion mit einer Dichte $d<1,006$ bzw. $d>1,006$. Die Mobilität ist auf die $\alpha$-Lipoproteinbande $(=1,0)$ bezogen. Trigly cerid-(TG) und Cholesterinkonzentration $(\mathrm{CH})$ in $\mathrm{mmol} / \mathrm{l}$ :

\begin{tabular}{|c|c|c|c|c|c|c|c|c|c|}
\hline & $\begin{array}{l}\text { nativ } \\
\text { TG }\end{array}$ & $\mathrm{CH}$ & $\begin{array}{l}\text { VLDL } \\
\text { TG }\end{array}$ & $\mathrm{CH}$ & $\begin{array}{l}\text { LDL } \\
\text { TG }\end{array}$ & $\mathrm{CH}$ & $\begin{array}{l}\text { HDL } \\
\text { TG }\end{array}$ & $\mathrm{CH}$ & $\begin{array}{l}\text { Mobi- } \\
\text { lität }\end{array}$ \\
\hline $\begin{array}{l}\text { a) } \\
\text { b) } \\
\text { c) } \\
\text { d) } \\
\text { e) } \\
\text { f) }\end{array}$ & $\begin{array}{l}4,67 \\
5,13 \\
3,98 \\
6,91 \\
2,58 \\
2,14\end{array}$ & $\begin{array}{r}9,97 \\
8,81 \\
8,55 \\
13,00 \\
7,33 \\
7,67\end{array}$ & $\begin{array}{l}2,98 \\
2,77 \\
2,82 \\
5,14 \\
0,64 \\
0,91\end{array}$ & $\begin{array}{l}3,81 \\
3,49 \\
5,34 \\
8,13 \\
1,22 \\
0,88\end{array}$ & $\begin{array}{l}1,11 \\
1,41 \\
0,67 \\
1,17 \\
1,33 \\
0,7 \dot{4}\end{array}$ & $\begin{array}{l}4,99 \\
4,17 \\
2,31 \\
3,52 \\
5,65 \\
5,69\end{array}$ & $\begin{array}{l}0,59 \\
0,94 \\
0,48 \\
0,48 \\
0,60 \\
0,38\end{array}$ & $\begin{array}{l}1,17 \\
1,34 \\
0,91 \\
1,35 \\
0,47 \\
1,09\end{array}$ & $\begin{array}{l}0,42 \\
0,53 \\
0,37 \\
0,35 \\
0,43 \\
0,47\end{array}$ \\
\hline
\end{tabular}

$\oplus$
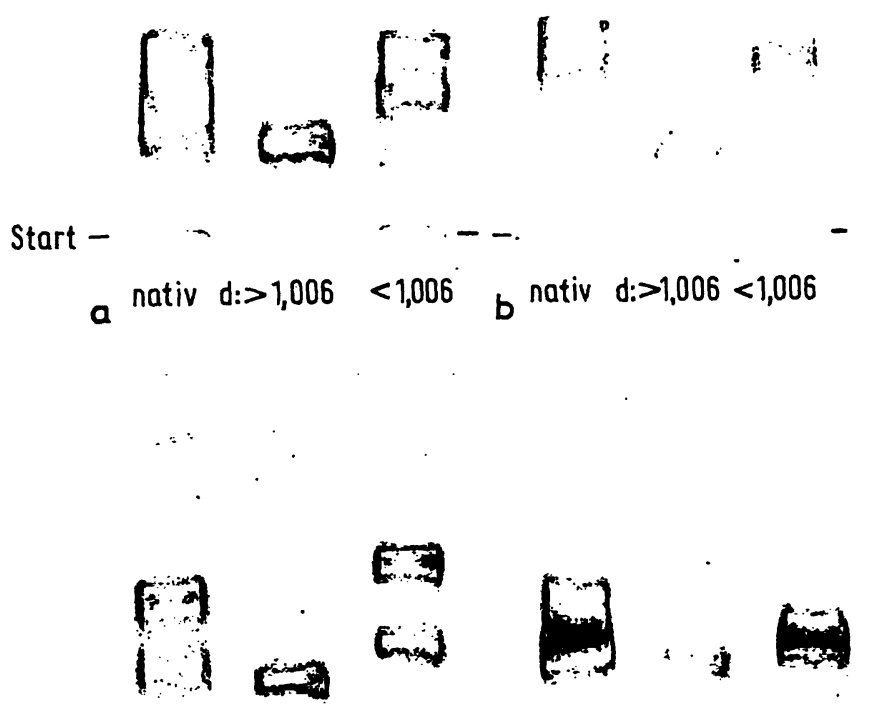

Start -

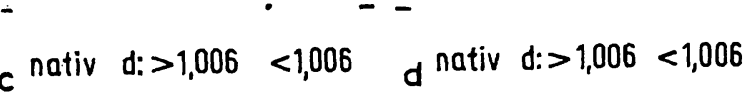

Tab. 2. Mittelwerte lipidchemischer Untersuchungskriterien (s. Text) und der elektrophoretischen Mobilität ( $\alpha$-Lipoproteinbande $=1,0$ ) von VLDL-Subfraktionen aus Seren $(n=106)$ von 42 untersuchten Patienten mit $(n=35)$ bzw. olune $(n=7)$ E-III Peptid der VLDL-Apolipoproteine. $\mathrm{TG}=$ Triglyceride, $\mathrm{CH}=$ Cholesterin.

\begin{tabular}{|c|c|c|c|c|c|}
\hline $\begin{array}{l}\text { VLDL- } \\
\text { Subfraktion }\end{array}$ & $\begin{array}{l}\text { Serum- } \\
\text { CH } \\
\text { Serum- } \\
\text { TG }\end{array}$ & $\begin{array}{l}\text { VLDL- } \\
\text { CH } \\
\text { Serum- } \\
\text { TG }\end{array}$ & $\begin{array}{l}\text { VLDL- } \\
\mathrm{CH} \\
\text { Serum- } \\
\mathrm{CH}\end{array}$ & $\begin{array}{l}\text { VLDL- } \\
\text { CH } \\
\text { VLDL- } \\
\text { TG }\end{array}$ & Mobilität \\
\hline $\begin{array}{l}\text { ohne Apo- } \\
\mathrm{E}_{\text {III }} \text {-Peptid }\end{array}$ & $\begin{array}{l}0,943 \pm \\
0,449\end{array}$ & $\begin{array}{l}0,425 \pm \\
0,209\end{array}$ & $\begin{array}{l}0,484 \pm \\
0,133\end{array}$ & $\begin{array}{l}0,594 \pm \\
0,250\end{array}$ & $\begin{array}{l}0,382 \pm \\
0,044\end{array}$ \\
\hline $\begin{array}{l}\text { mit Apo- } \\
\text { E }_{\text {III-Peptid }}\end{array}$ & $\begin{array}{l}1,267 \pm \\
0,733\end{array}$ & $\begin{array}{l}0,237 \pm \\
0,127\end{array}$ & $\begin{array}{l}0,245 \pm \\
0,164\end{array}$ & $\begin{array}{l}0,353 \pm \\
0,199\end{array}$ & $\begin{array}{l}0,485 \pm \\
0,065\end{array}$ \\
\hline $\begin{array}{l}t= \\
p<\end{array}$ & $\begin{array}{l}1,805 \\
0,05\end{array}$ & $\begin{array}{l}5,222 \\
0,001\end{array}$ & $\begin{array}{l}5,978 \\
0,001\end{array}$ & $\begin{array}{l}4,550 \\
0,001\end{array}$ & $\begin{array}{l}7,355 \\
0,001\end{array}$ \\
\hline
\end{tabular}

Zeichen diagnostisch bedeutsam. Versuche, ihre diagnostische Bedeutung durch verbesserte Bestimmungsmethoden zu vergrößern $(3,12)$, werden leicht überbewertet. Da „Ausnahmen “ der zugrunde liegenden Normierung nicht auszuschließen sind, sollte die Diagnose zumindest von mehreren indirekten Kriterien abhängig gemacht werden.

Die spezifische Bedeutung für das Vorkommen verschiedener VLDL-Subfraktionen ist unbekannt. Havel et al. (13) grenzten ein ,slow" prä- $\beta$-VLDL von $\beta$-VLDL ab. Nach Meinung der Autoren kann diese Subfraktion als „intermediate density lipoprotein “ in normo- wie hyperlipämischen Seren vorkommen. Carlson \& Carlson (14) fanden bei $25-30 \%$ von 609 untersuchten Seren eine VLDL-Subfraktion, die aufgrund einer größeren elektrophoretischen Mobilität von $\beta$-VLDL zu unterscheiden war. Sie bezeichneten die Subfraktion als ,late migrating“ prä-ß-Lipoprotein, zeigten jedoch, daß der VLDLCholesterin/VLDL-Triglycerid Quotient dieser Seren wie bei Typ III Hyperlipoproteinämien erhöht ist und daß außerdem der Triglyceridgehalt in LDL und HDL, vergleichbar den vorliegenden Befunden bei diesen Seren mit einer VLDL-Subfraktion größer ist als bei Kontrollen ohne diese Fraktion. Hyperlipoproteinämien mit prä- $\beta$-VLLL-Subfraktionen könnten deshalb verwandte Formen einer heterozygot vererbten Typ III Hyperlipoproteinämie darstellen.

\section{Danksagung}

Start -

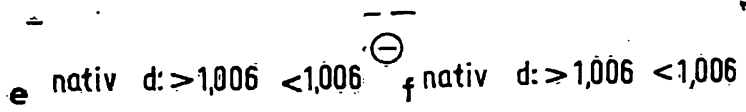

Frau Barbara Heger sei an dieser Stelle für ihre wertvolle technische Mitarbeit Dank gesagt.

\section{Literaturverzeichnis}

1. Bèaumont, J. L., Carlson, L., Cooper, R. G., Fejfar, Z., Fredrickson, D. \& Strasser, T. (1970), Bull. WHO 43, $891 \rightarrow 915$

2. Mishkel, M. A., Nazir, D. J. \& Crwother, S. (1975), Clin. Chem. Acta 58, 121-136.

3. Vessby, B. (1976), Clin. Chem. Acta 69, 29-42.

4. Seidel, D. \& Greten, H. (1970), Clin. Chem. Acta 30, $31-36$.

5. Wieland, H. \& Seidel, D. (1973), Clin. Chem. 19, $1139-1141$. 
6. Quarfordt, S., Levy, R. \& Fredrickson, D. S. (1971), J. Clin. Invest. 50, 754-761.

7. Patsch, J. R., Sailer, S. \& Braunsteiner, H. (1975), Europ. J. Clin. Invest. 5, 45-55.

8. Utermann, G., Jaeschke, M. \& Menzel, J. (1976), FEBS Letters 56, 352-355.

9. Vogelberg, K. H., Utermann, G. \& Gries, F. A. (1973), dieses J. 11, 291-296.

10. Hazzard, W. R., Porte Jr., D. \& Biermann, E. L. (1973), Metabolism 21,1009-1019.
11. Fredrickson, D. S., Morganroth, J. \& Levyy, R. (1975), Ann. Intern. Med. 82, 150-157.

12. Albers, J. J., Russe! Warnick, G., Hazżard, W. R. (1977), Clin. Chim. Acta 75, 193=204.

13. Havel, R. J., Kane, J. P. \& Pagnan, A. (1976), in: Lipoprotein mețabolism (H. Greten, 4., ed.), Springer-Verlag Berlin, Heidelberg, New York, S. 65-68.

14. Carlson, K. \& Carlsọn, L. A. (1975), Scand. J. Clin. Lab. Invest. 35, 655-660.

PD́ Dr. Karl Heinrich Vogelberg Auf'm Hennekamp 65

Diabetes-Forschungsinstitut

an der Universität

D-4000 Düsseldorf 1 